\title{
Support Group Intervention Reduces Social Alienation and Loneliness among College Students with Victimization Experience
}

\section{Cut Maghfirah Faisal ${ }^{\mathrm{a}}$ and Sherly Saragih Turnip ${ }^{\mathrm{b}}$}

${ }^{a}$ Faculty of Psychology, Universitas Indonesia, Depok, Indonesia; ${ }^{b}$ Department of Clinical Psychology, Faculty of Psychology, Universitas Indonesia, Depok, Indonesia

*Corresponding author:

Sherly Saragih Turnip

Department of Clinical Psychology

Faculty of Psychology, Universitas Indonesia

Jl. Lkr. Kampus Raya, Depok, Jawa Barat

Indonesia, 16424

Tel.: +62 217270004

Email address: sherly.saragih@ui.ac.id 


\title{
Support Group Intervention Reduces Social Alienation and Loneliness among College Students with Victimization Experience
}

\begin{abstract}
Victimization can have long-term effects, including bringing about a state of social alienation or loneliness. People with social alienation or loneliness may have difficulty initiating and maintaining social relationships, which then exacerbates the alienation and loneliness. If it is left untreated, other effects could result, such as depression, substance abuse, vandalism, and poor health. An intervention is needed to reduce the symptoms of social alienation and loneliness among the victimized. Several studies have shown that support groups can reduce such symptoms and that they help broaden social networks as well. This study tested the effectiveness of support group intervention program in reducing the symptoms of social alienation and loneliness among college students who have been victimized. This was a short program, consisting of only three sessions. During this program, participants were encouraged to talk about their negative social experiences and the difficulty they encounter in maintaining social relationships. They learned useful tips and skills to improve their interpersonal relationships, and communication skills. Certain methods were featured in this program, such as roleplay, group interview, snowball feedback, and home assignments. Eight college students aged 18 to 22 were selected to join the program. All of them had experienced victimization, including verbal, physical, and cyber bullying, as well as estrangement from friends and families. They also reported difficulties in interpersonal relationships due to the victimization. Six participants attended all sessions, and two could not. This study had a pre- and post-test, withingroup design. To measure the degree of social alienation and loneliness among the participants, we used the Jessor and Jessor Social Alienation Scale and the De Jong Gierveld Loneliness Scale. Both scales were administered before the first session and immediately following the last one. The scores from the pre- and post-tests were compared using descriptive statistics to evaluate the effectiveness of the program. Qualitative evaluations were also collected from all participants to describe changes among them after they joined the program. The pre- and post-test measurements revealed that all participants had reduced scores on the social alienation scale from the pre- to the post-test. On the loneliness scale, one participant had an increased score on the post-test, while the rest of the participants showed a decrease. This may have been the result of certain personal characteristics of this participant. Qualitative evaluations indicated that all participants had positive change in their social relationships and the way they perceived themselves after the program.
\end{abstract}

Keywords: support group, social alienation, loneliness, victimization, college students

\section{Introduction}

Negative social experiences, such as victimization, can have prolonged effects. In victimization, a person becomes the victim of unfair and undelightful treatment (Rudolph et al., 2014). Victimization can occur in various forms, including physical victimization (e.g., being beaten), verbal victimization (e.g., being mocked), and relational victimization (e.g., estrangement) (Rudolph et al., 2014). Victimization at school could predict social alienation and loneliness in adolescence and adulthood (Rudolph et al., 2014). Low social status and harmful friendships 
resulting from victimization can estrange victims from their social circle. This estrangement can be reflected by loneliness and social dissatisfaction, as well as social helplessness, as expressed in avoiding social situations (Rudolph et al., 2014). This avoidance behavior could in turn worsen the social alienation and loneliness became worse.

Social alienation can have several negative effects. A person with social alienation has a greater risk of acquiring deviant peer affiliations (Rudolph et al., 2014). Additionally, teenage social alienation can result in deviant behavior, such as reckless sexual relationships, violence, vandalism, absenteeism, and substance abuse (Safipour, Tessma, Higginbottom, \& Emami, 2010).

Like social alienation, loneliness can have negative impacts. Lonely individuals are more prone to depression, loneliness can affect physical health, and People with loneliness are more vulnerable to viruses and sleep disturbances (Cacioppo \& Hawkley, 2009).

Studies have found that social alienation and loneliness are strongly correlated with deficits in social skills (Lodder, Goossens, Scholte, Engels, \& Verhagen, 2016). People with social alienation and loneliness use less effective strategies in interaction (Jones, Hobbs, \& Hockenbury, 1982). They attempt to withdraw from their surroundings, making their symptoms worse (Rudolph et al., 2014). Therefore, it may be beneficial for such individuals to study effective social skills.

People with social alienation and loneliness is their self-depreciation (Lasgaard \& Elklit, 2009) and often have negative self-evaluation. For this reason, intervention for them could also target their self-depreciation.

A support group is a useful intervention for reducing social isolation and loneliness (Windle, Francis, \& Coomber, 2011), where it can be designed according to the features and needs of people with social alienation and loneliness. The main goal of such a support group is to support victims as they attempt to widen their social network. In a support group, victimized individuals can learn how to interact with others. They also have a chance to share their stories and their symptoms of social alienation and loneliness. Such opportunities are important to alienated individuals, as they tend to avoid talking about their problems. After joining their support group program, they could also learn from each other's experiences (Yalom, 2005). They could be guided to evaluate potential solutions for their particular issues.

Support group intervention has been found to be useful in reducing social alienation and loneliness (Windle, Francis, \& Coomber, 2011), but most support groups are designed for more than six sessions and longer than six months (Medical Advisory Secretariat, 2008). This study had several limitations, such as its potentially high rate of absenteeism, which is especially prevalent in the college student population it drew from. Therefore, this study used a shortduration support group intervention that had the potential to be beneficial for certain populations. 


\section{Methods}

\section{A. Data Collection}

We conducted an online survey to select eligible participants for this support group program. The inclusion criteria were college students aged 18 to 22 who had been victimized. The survey was completed by 43 college students, and 14 students had social alienation or loneliness scores within the inclusion criteria. Then, an individual interview was conducted, After the interview, we selected eight participants to join the support group. All of the selected participants had experienced victimization, including verbal, physical, and cyber bullying, as well as estrangement from friends and families. They also reported difficulties in interpersonal relationships due to their victimization. We collected informed consent from all selected participants, and all were informed of their right to withdraw from the program at any time without consequence.

\section{B. Research Design}

This study employed a pre- and post-test, within-group design. We measured social alienation and loneliness among the same group twice: once before the first session of the support group program (pre-test) and again after the last session of the program (post-test). The results of those measurements were then compared to deduce the effectiveness of the support group program for reducing social alienation and loneliness.

\section{Measures}

We used the Jessor and Jessor Social Alienation Scale (Jessor \& Jessor, 1977) to measure social alienation among the participants. This scale measures general alienation in relation to ambiguity of role and daily activity, as well as the belief that the alienated person was isolated from others. This scale consists of 15 items, measured on a four-point Likert scale, with responses of "(1) Strongly agree," “(2) Agree," "(3) Disagree,” and “(4) Strongly disagree.” The possible scores range between 15 (low alienation) to 60 (high alienation).

To measure loneliness, we used the De Jong Gierveld Loneliness Scale (2006). This scale was developed from the loneliness theory created by Weiss (1973), which states that loneliness comes in two types: social loneliness and emotional loneliness. The De Jong Gierveld Loneliness Scale uses a four-point Likert scale: "(1) Strongly disagree," "(2) Disagree," "(3) Agree," and "(4) Strongly agree." The possible total score obtained from this scale ranged from 0 to 6 . Higher scores reflect greater loneliness.

\section{Data analysis}

All the participants' scores for social alienation and loneliness were compared using descriptive statistics to evaluate the effectiveness of the support group program in reducing social alienation and loneliness. Qualitative evaluations from all participants were also collected to describe the changes they witnessed in themselves after going through the program. 


\section{E. Support group program}

The support group program consisted of three sessions. Each session lasted for 120 minutes. In the first, the participants were prompted to talk about their victimization experience and about their symptoms of social alienation and loneliness. This accentuate the assertion that the participants were not alone, and it encouraged them to open themselves to the other participants more fully. They were also asked to share solutions that they had already attempted.

The main agenda of the second session was to practicing the communication skills of all participants; this was an important agenda, as all participants considered themselves to have poor ability to initiate and maintain communication with others. The participants were broken into pairs. They were instructed to interview each other about their daily activities and hobbies. After they had completed their paired interview, all participants were asked to tell their partner about their impressions. Thus, the participants were able to work on communication skill, and they also had the chance to improve their self-worth. At the end of the session, all participants were given homework, namely, to initiate communication with someone with whom they felt it would be difficult to talk.

The participants began the third session by sharing the result they obtained from their homework. Following that, we proceeded to the main agenda: roleplay. The main purpose of the roleplay was to give the participants room to learn how to face uncomfortable social situations. Several scenarios were prepared by the facilitator, such as bumping into an old friend who may not remember the participant and feeling ignored by others in a conversation. Each participant played different parts in the roleplays. After each scenario had been performed each all participants was asked to evaluate the responses used by the performers. Thus, they could learn adaptive and useful strategies from each other that they could use to face uncomfortable social situations.

Because all participants had been the victim of at least one negative social experience, it was considered likely that they would feel uncomfortable discussing their situation. The facilitator, therefore, was required to master some emotional stability techniques, such as progressive relaxation and point-of-power. Those tools were very useful for calming the participants in instances of high stress.

In this program, we featured several informal methods, such as the snowball and love letter. The snowball method was used in the second session. Each participant anonymously wrote their impressions of the others. Then, the love letter method was featured in the last session. The participants were free to write down any impression, suggestion, or motivation to all other participants. These informal methods were useful for breaking the ice and enhancing the participants' self-worth, as almost all participants considered themselves worthless compared to the others. 


\section{Results}

\section{A. Demographic Data}

Eight participants were invited to join the program. They were undergraduate students in different departments of the University of Indonesia who fulfilled all inclusion criteria. The demographic data for all participants are presented in Table 1.

Table I. Demographic Data of Participants

\begin{tabular}{|c|c|c|c|}
\hline Participant & Gender & Age & Victimization Experience \\
\hline A & F & 19 & Bullying (verbal, physical, cyber-) \\
\hline B & F & 21 & Bullying (verbal) \\
\hline C & F & 21 & Bullying (verbal, physical) \\
\hline D & F & 21 & Bullying (verbal, physical, cyber-) \\
\hline E & F & 22 & Bullying (verbal, physical) \\
\hline F & M & 21 & Bullying (verbal) \\
\hline G & F & 18 & Estranged from friends and mother \\
\hline H & M & 20 & Bullying (verbal, physical, cyber-) \\
\hline
\end{tabular}

\section{B. Change of Social Alienation and Loneliness Score}

All eight participants managed to complete the pre-test, while only six participants took the post-test. The pre- and post-test results of all participants are presented in Table 2 .

Table II. Pre- and Post-Test Results

\begin{tabular}{|l|l|l|l|l|}
\hline \multirow{2}{*}{ Participant } & \multicolumn{2}{l|}{ Social Alienation } & \multicolumn{2}{l|}{ Loneliness } \\
\cline { 2 - 5 } & Pre-Test & Post-Test & Pre-Test & Post-Test \\
\hline A & 45 & & 4 & - \\
\hline B & 41 & 35 & 4 & 3 \\
\hline C & 45 & 34 & 6 & 1 \\
\hline D & 47 & 44 & 3 & 4 \\
\hline E & 43 & 36 & 5 & 2 \\
\hline F & 44 & 37 & 5 & 2 \\
\hline G & 41 & 37 & 5 & 2 \\
\hline H & 45 & & 4 & - \\
\hline
\end{tabular}

The change in the social alienation and loneliness scores from pre- to post-test is presented in Figs. 1 and 2. 


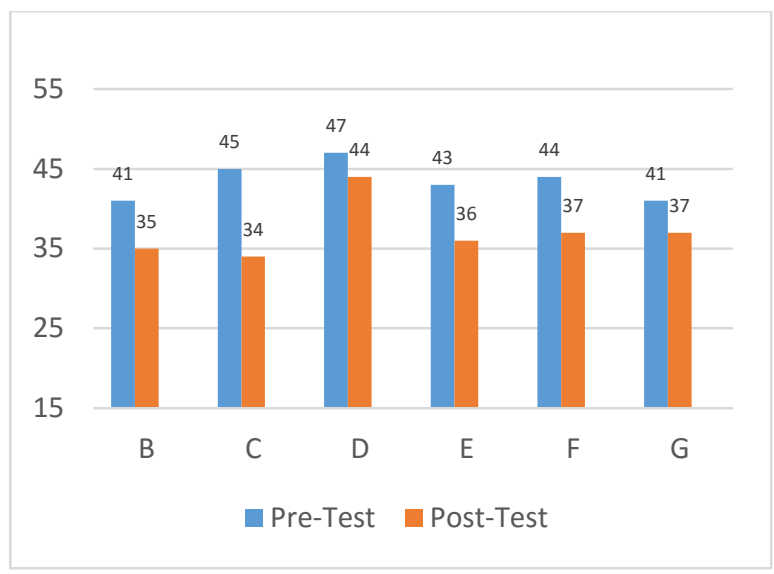

Fig. 1. Change in Social Alienation Score

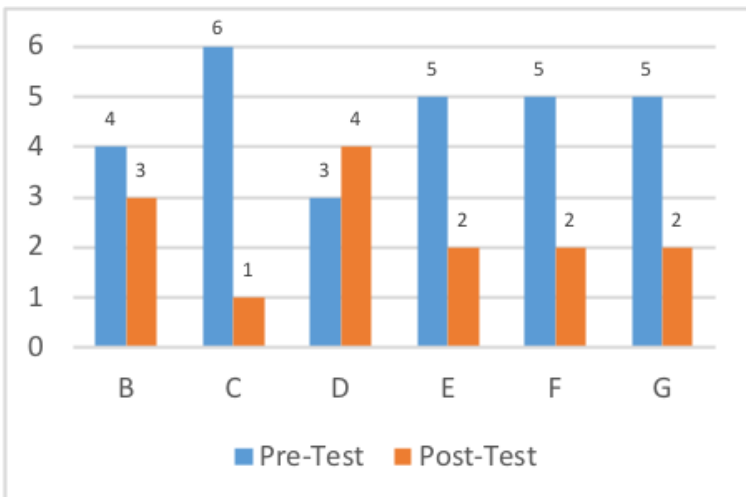

Fig. 2. Change in Loneliness Score

These measurements indicate that all participants' social alienation symptoms declined after they participated in the support group program. All but one participant reported fewer loneliness symptoms.

\section{Discussion and Conclusion}

In general, all participants showed reduced scores for social alienation and loneliness after participating in the support group program. As mentioned previously, social alienation and loneliness were strongly correlated with social skill deficits (Lodder et al., 2016). Lonely and alienated individuals use less effective social strategies (Jones, Hobbs \& Hockenbury, 1982). To overcome the program enabled the participants to learn several useful social skills, including basic communication skills and the skills needed to face uncomfortable social situations. Such skills were proven to be useful in participants' life, which was indicate by the reduced scores for social alienation and loneliness after participating in the program.

Lonely and alienated people often have low self-esteem and tend toward self-depreciation (Lasgaard \& Elklit, 2016). These symptoms were treated on the second session, in which participants were paired and interviewed each other, after which they were asked to inform the group of the positive impressions they had of their partner. The feedback from their partner helped the participants to perceive their own positive sides of them and helped them appreciate 
themselves more. This was another useful method for reducing social alienation and loneliness, targeting their self-depreciation.

Some participants experienced larger symptom reductions than others. Others reported minimal changes or even loneliness scores. This may imply that the support group may not have fit the needs and conditions of all participants. In relation to the finding that social alienation and loneliness are correlated with social skill deficits (Lodder et al., 2016), it is reasonable to argue that such programs would suit those with poor social and communication skills more. In addition, certain personal characteristics of the participants may also affect their degree of development, such as motivation, cognitive abilities, severity of symptoms, and level of insight (Lynch, 2012).

Participant D reported the least change in the social alienation score and even an increased level of loneliness. We argue that D's resistance and lack of motivation to change was the cause of this. D claimed that she was comfortable with her alienation. This state was very different from that of the other participants, who felt extremely uncomfortable with their alienation and loneliness. D also stated that she already knew and practiced the communication solutions focused on by the program. The facilitator observed that D did have better social and communication skills than the others. Hence, we can conclude that the methods and solutions featured in this support group program were not suitable to D's symptoms, resulting in no significant change in symptoms.

Several evaluations need to be performed before the next implementation of this program. The facilitator needs to examine the characteristics of the participants before they are selected to join the program. The people who would gain maximum benefit from the program are motivated, insightful, feel uncomfortable with their alienation or loneliness, and have poor social and communication skills (Lodder et al., 2016; Lynch, 2012). The participants' commitment to and availability for participating in all sessions should also receive notice.

While a similar support group program is being run, the facilitator must be aware of the participants' moments of emotional stress, such as crying while describing their victimization. Hence, it would be helpful to prepare some useful tools, such as tissue, water, and more importantly, emotion stabilization techniques to demonstrate to the participants. This technique can be used at the beginning and end of each session, along with when participants have emotional reactions.

\section{Acknowledgement}

The authors thank all the participants in this program and the staff of the Faculty of Psychology University of Indonesia, who provided facilities for the support group program. 


\section{References}

Cacioppo, J. T., \& Hawkley, L. C. (2009). Perceived social isolation and cognition. Trend in Cognitive Sciences, 13(10), 447-454.

Gierveld, J. D. J., \& Van Tilburg, T. (2006). A 6-item scale for overall, emotional, and social loneliness: Confirmatory tests on survey data. Research on Aging, 28(5), 582-598.

Jessor, R., \& Jessor, S. (1977). Problem Behavior and Psychosocial Development. New York, NY: Academic Press.

Jones, W. H., Hobbs, S. A., \& Hockenbury, D. (1982). Loneliness and social skill deficits. Journal of Personality and Social Psychology, 42(4), 682-689.

Lasgaard, M., \& Elklit, A. (2009). Prototypic features of loneliness in a stratified sample of adolescents. Interpersona: An International Journal on Personal Relationships, 33, 85-110.

Lynch, M. M. (2012). Factor influencing successful psychotherapy outcomes. St. Catherine University, Minnesota. Retrieved from

https://sophia.stkate.edu/cgi/viewcontent.cgi?referer=https://scholar.google.co.id/\&httpsredir=1\&article= $1057 \&$ context=msw_papers

Lodder, G. M. A., Goossens, L., Scholte, R. H. J., Engels, R. C. M. E., \& Verhagen, M. (2016). Adolescent loneliness and social skills: Agreement and discrepancies between self-, meta-, and peer-evalutions. Journal of Youth and Adolescence, 45(12), 2406-2416.

Medical Advisory Secretariat. (2008). Social isolation in community-dwelling seniors: An evidence-based analysis. Ontario Health Technology Assessment Series. 8.

Rudolph, K. D., Lansford, J. E., Agoston, A. M., Sugimura, N., Schwartz, D., Dodge, K. A., ... Bates, J, E, (2014). Peer victimization and social alienation: Predicting deviant peer affiliation in middle school. Child Development, 85(1), 124-139.

Safipour, J., Tessma, M. K., Higginbottom, G., \& Emami, A. (2010). Measuring social alienation in adolescence: Translation and validation of Jessor and Jessor Social Alienation Scale. Scandinavian Journal of Psychology.,51(6), 517-524.

Weiss, R. S. (1973). Loneliness: The Experience of Emotional and Social Isolation. Cambridge, MA: MIT Express.

Windle, K., Francis, J., \& Coomber, C. (2011). Preventing Social Isolation and Cognition: Interventions and Outcomes. London: Social Care Institute of Excellence.

Yalom, D. (2005). The Theory and Practice of Group Psychotherapy. New York, NY: Basic Books. 